\title{
A MULTIPLICATIVE PROPERTY OF QUANTUM FLAG MINORS II
}

\author{
PHILIPPE CALDERO AND ROBERT MARSH
}

\begin{abstract}
Let $U^{+}$be the plus part of the quantized enveloping algebra of a simple Lie algebra and let $\mathcal{B}^{*}$ be the dual canonical basis of $U^{+}$. Let $b, b^{\prime}$ be in $\mathcal{B}^{*}$ and suppose that one of the two elements is a $q$-commuting product of quantum flag minors. We show that $b$ and $b^{\prime}$ are multiplicative if and only if they $q$-commute.
\end{abstract}

\section{Contents}

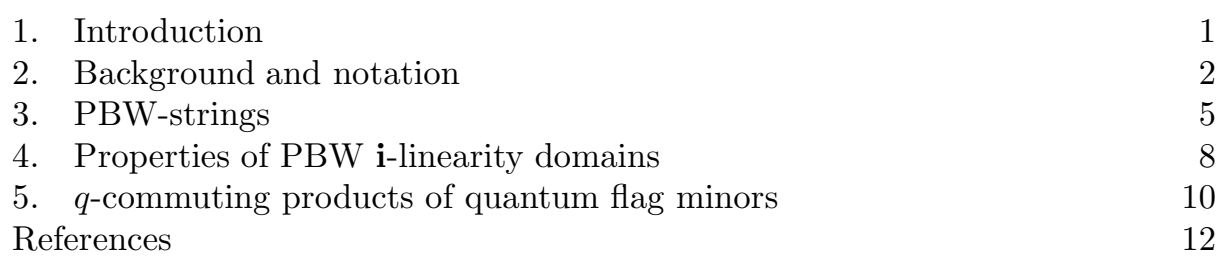

\section{INTRODUCTION}

Let $\mathcal{B}^{*}$ denote the dual of the canonical basis [8, 14, $\$ 14.4 .6$ ] of a quantized enveloping algebra of a simple Lie algebra $\mathfrak{g}$. Two elements of $\mathcal{B}^{*}$ are said to be multiplicative if their product also lies in $\mathcal{B}^{*}$ up to a power of $q$. They are said to $q$-commute if they commute up to a power of $q$. The Berenstein-Zelevinsky conjecture states that two elements of $\mathcal{B}^{*}$ are multiplicative if and only if they $q$ commute. While Reineke [17, 4.5] has shown that if two elements are multiplicative then they $q$-commute, the conjecture is now known to be false: a counter-example was provided by Leclerc [10, who showed that for all but finitely many types of simple Lie algebra, there exist elements $b \in \mathcal{B}^{*}$ whose square does not lie in $\mathcal{B}^{*}$ even up to a power of $q$ (such elements obviously $q$-commute with themselves). Such elements are called imaginary; elements of $\mathcal{B}^{*}$ whose square does lie in $\mathcal{B}^{*}$ up to a power of $q$ are known as real. We consider the following question:

Question 1.1. Let $b, b^{\prime}$ be in $\mathcal{B}^{*}$ and suppose that $b$ is real. Is it the case that $b$ and $b^{\prime}$ are multiplicative if and only if they $q$-commute?

Date: 24th January 2003.

The authors would like to thank EC grant of the TMR network "Algebraic Lie Representations", contract no. ERB FMTX-CT97-0100, for support of the visit of both authors to Wuppertal in October 2001 and for support of the first author's visit to Leicester in August 2002, and the University of Leicester for study leave for the second author in Autumn 2002. 
Leclerc has made a conjecture [10, Conjecture 1] concerning the expansion of the product of two dual canonical basis elements, and has remarked that if true, it would imply that the answer to this question is yes in general. In this paper, we prove that the answer to this question is yes if one of the two elements involved is a $q$ commuting product of quantum flag minors (in type $A$ ). We note that such products are known to be real [11. A key part of our proof involves results concerning the piecewise-linear reparametrization function $R_{\mathbf{i}}^{\mathbf{i}^{\prime}}$ associated by Lusztig to a pair $\mathbf{i}, \mathbf{i}^{\prime}$ of reduced decompositions for the longest word $w_{0}$ in the Weyl group of $\mathfrak{g}$. Lusztig has defined the canonical basis via the bases of Poincaré-Birkhoff-Witt type associated to such reduced decompositions, and such reparametrization functions arise from taking two of the Lusztig parametrizations of the canonical basis. We show that these functions share some of the properties known to be possessed by the reparametrization functions arising from string parametrizations for the canonical basis 1. In particular we show that if two dual canonical basis elements $q$-commute, then their PBW parametrizations (with respect to a fixed reduced decomposition i) lie in a single PBW $\mathbf{i}$-linearity domain; in other words, if their parameters are $\mathbf{m}$ and $\mathbf{m}^{\prime}$, then $R_{\mathbf{i}}^{\mathbf{i}^{\prime}}\left(\mathbf{m}+\mathbf{m}^{\prime}\right)=R_{\mathbf{i}}^{\mathbf{i}^{\prime}}(\mathbf{m})+R_{\mathbf{i}}^{\mathbf{i}^{\prime}}\left(\mathbf{m}^{\prime}\right)$ for all reduced decompositions $\mathbf{i}^{\prime}$ for $w_{0}$. The corresponding result for string parametrizations is already known [1, 2.9]. Such behaviour hints at an explanation for the compatibility of examples in which the canonical basis has been computed explicitly [6], [15], 21] with respect to linearity domains. As a consequence, we obtain that the set of PBW i-linearity domains forms a fan in $\mathbb{R}^{N}$, where $N$ is the length of $w_{0}$.

This enables us to prove that the answer to Question 1.1 is yes in type $\mathrm{A}_{n}$ when $b$ is a $q$-commuting product of quantum flag minors. This generalizes a theorem of $[\underline{5}$.

In the paper, the Lie algebra $\mathfrak{g}$ is supposed to be of type $\mathrm{A}_{n}$. Note that results in section 1-4 can be easily generalized to all simply-laced types. Note also that Lemma 5.3 is only true in type $\mathrm{A}_{n}$. Hence, Theorem [5.5 needs this assumption.

\section{BACKGROUND AND NOTATION}

We use the set-up of 4 . Let $\mathfrak{g}=s l_{n+1}(\mathbb{C})$ denote the simple Lie algebra of type $A_{n}$. Let $\mathfrak{h}$ be a Cartan subalgebra and let $\mathfrak{g}=\mathfrak{n}^{-} \oplus \mathfrak{h} \oplus \mathfrak{n}^{+}$be a compatible triangular decomposition. Let $\alpha_{1}, \alpha_{2}, \ldots, \alpha_{n}$ be the corresponding simple roots, and $\Delta^{+}$the corresponding set of positive roots. Let $W$ be the Weyl group associated to the root system, $P$ be the weight lattice generated by the fundamental weights $\varpi_{i}, 1 \leq i \leq n$, and $\langle$,$\rangle be the W$-invariant form on $P$. Let $U=U_{q}(\mathfrak{g})$ be the simply connected Drinfel'd-Jimbo quantized enveloping algebra over $\mathbb{Q}(q)$ associated to $\mathfrak{g}$ as defined in [7. Let $U^{-}, U^{0}$ and $U^{+}$be the subalgebras associated to the sub-Lie algebras $\mathfrak{n}^{-}$, $\mathfrak{h}$ and $\mathfrak{n}^{+}$respectively; we have the triangular decomposition $U \cong U^{-} \otimes U^{0} \otimes U^{+}$. The subalgebra $U^{+}$is generated over $\mathbb{Q}(q)$ by canonical generators $E_{1}, E_{2}, \ldots E_{n}$, subject to the quantized Serre relations; the subalgebra $U^{-}$is isomorphic to $U^{+}$, with corresponding generators $F_{1}, F_{2}, \ldots F_{n}$, and the subalgebra $U^{0}$ is isomorphic to $\mathbb{Q}(q)[P]$, the element corresponding to $\lambda \in P$ being denoted by $K_{\lambda}$. Let $\mathfrak{b}^{+}=\mathfrak{h} \oplus \mathfrak{n}^{+}$ and $\mathfrak{b}^{-}=\mathfrak{h} \oplus \mathfrak{n}^{-}$and we define $U\left(\mathfrak{b}^{+}\right)=U^{0} U^{+}$and $U\left(\mathfrak{b}^{-}\right)=U^{-} U^{0}$. The algebra $U$ is a Hopf algebra with comultiplication $\Delta$, antipode $S$ and augmentation $\varepsilon$ given by:

$$
\Delta\left(E_{i}\right)=E_{i} \otimes 1+K_{\alpha_{i}} \otimes E_{i}, \quad \Delta\left(F_{i}\right)=F_{i} \otimes K_{-\alpha_{i}}+1 \otimes F_{i}, \quad \Delta\left(K_{\lambda}\right)=K_{\lambda} \otimes K_{\lambda},
$$




$$
\begin{gathered}
S\left(E_{i}\right)=-K_{-2 \alpha_{i}} E_{i}, \quad S\left(F_{i}\right)=-F_{i} K_{2 \alpha_{i}}, \quad S\left(K_{\lambda}\right)=K_{-\lambda}, \\
\varepsilon\left(E_{i}\right)=\varepsilon\left(F_{i}\right)=0, \quad \varepsilon\left(K_{\lambda}\right)=1 .
\end{gathered}
$$

The root lattice $Q$ is defined to be $Q=\sum_{i} \mathbb{Z} \alpha_{i}$, with $Q^{+}=\sum_{i} \mathbb{Z}_{\geq 0} \alpha_{i}$. Recall that if $\alpha=\sum_{i} m_{i} \alpha_{i} \in Q^{+}$, then an element in the subspace of $U$ generated by $\left\{E_{i_{1}}^{n_{1}} E_{i_{2}}^{n_{2}} \cdots E_{i_{k}}^{n_{k}}: n_{1} \alpha_{i_{1}}+n_{2} \alpha_{i_{2}}+\cdots+n_{k} \alpha_{i_{k}}=\alpha\right\}$ (respectively, $\left\{F_{i_{1}}^{n_{1}} F_{i_{2}}^{n_{2}} \cdots F_{i_{k}}^{n_{k}}:\right.$ $\left.n_{1} \alpha_{i_{1}}+n_{2} \alpha_{i_{2}}+\cdots+n_{k} \alpha_{i_{k}}=\alpha\right\}$ ), is said to have weight $\alpha$ (respectively, $-\alpha$ ). For all $\alpha \in Q^{+}$, let $U_{\alpha}^{+}$(respectively, $U_{-\alpha}^{-}$) denote the subspace of $U_{+}$(respectively, $U^{-}$) consisting of elements of weight $\alpha$ (respectively, $-\alpha$ ). We write the weight of an element $X$ as $w t(X)$ (if it exists), and $\operatorname{tr}(X)$ (or $\operatorname{tr}(w t(X))$ ) for the sum $\sum_{i} m_{i}$ if $w t(X)=\sum_{i} m_{i} \alpha_{i}$. For $u \in U$, set $\Delta(u)=u_{(1)} \otimes u_{(2)} \in U \otimes U$. There exists a unique bilinear form $($,$) [18, 20] on U\left(\mathfrak{b}^{+}\right) \times U\left(\mathfrak{b}^{-}\right)$satisfying

$$
\begin{array}{cc}
\left(E_{i}, F_{i}\right)=\delta_{i j}\left(1-q^{2}\right)^{-1}, \\
\left(u^{+}, u_{1}^{-} u_{2}^{-}\right)=\left(\Delta\left(u^{+}\right), u_{1}^{-} \otimes u_{2}^{-}\right), & u^{+} \in U\left(\mathfrak{b}^{+}\right), u_{1}^{-}, u_{2}^{-} \in U\left(\mathfrak{b}^{-}\right), \\
\left(u_{1}^{+} u_{2}^{+}, u^{-}\right)=\left(u_{2}^{+} \otimes u_{1}^{+}, \Delta\left(u^{-}\right)\right), & u^{-} \in U\left(\mathfrak{b}^{-}\right), u_{1}^{+}, u_{2}^{+} \in U\left(\mathfrak{b}^{+}\right), \\
\left(K_{\lambda}, K_{\mu}\right)=q^{-(\lambda, \mu)}, \quad\left(K_{\lambda}, F_{i}\right)=0, & \left(E_{i}, K_{\lambda}\right)=0, \quad \lambda, \mu \in P .
\end{array}
$$

The form $\left(\right.$, ) is nondegenerate on $U_{\alpha}^{+} \otimes U_{-\alpha}^{-}$for all $\alpha \in Q_{+}$. Since $U^{+}$and $U^{-}$are isomorphic algebras (with isomorphism preserving their weight spaces), we can naturally identify $U_{-\alpha}^{-}$with $U_{\alpha}^{+}$, so for each element $u \in U_{\alpha}^{+}$there is a corresponding element $u^{*} \in U_{\alpha}^{+}$(corresponding to $u$ using the form). Let $w_{0}$ be the longest element of $W$; denote by $R\left(w_{0}\right)$ the set of all reduced decompositions for $w_{0}$. Fix a reduced decomposition $\mathbf{i}=\left(i_{1}, i_{2}, \ldots, i_{N}\right)$ of $w_{0}$, and for $1 \leq t \leq N$, let $\beta_{t}=s_{i_{1}} s_{i_{2}} \cdots s_{i_{t-1}}\left(\alpha_{i_{t}}\right)$; we get an ordering $\beta_{1}<\beta_{2}<\cdots<\beta_{N}$ of $\Delta^{+}$. For $1 \leq i \leq n$, let $T_{i}$ denote the Lusztig braid automorphism of $U^{+}$[14 37.1.3], 19] associated to $i$. For $1 \leq t \leq N$ let $E_{\beta_{t}}=T_{i_{1}} T_{i_{2}} \cdots T_{i_{t-1}}\left(E_{i_{t}}\right)$. The PoincaréBirkhoff-Witt basis of $U^{+}$is the basis

$$
B_{\mathbf{i}}=\left\{E(\mathbf{m}): \mathbf{m} \in \mathbb{Z}_{\geq 0}^{N}\right\},
$$

where

$$
E(\mathbf{m})=E_{\mathbf{i}}(\mathbf{m})=\prod_{t=1}^{N} \frac{1}{\left[m_{t}\right] !} E_{\beta_{t}}^{m_{t}},
$$

with the product taken in the ordering given above. Here $[m] !=[m][m-1] \cdots[1]$, where $[m]=\frac{q^{m}-q^{-m}}{q-q^{-1}}$. By [12] we have that

$$
E(\mathbf{m})^{*}=\prod_{t=1}^{N} \psi_{m_{t}}\left(q^{2}\right) E(\mathbf{m})
$$

where $\psi_{m}(z)=\prod_{k=1}^{m}\left(1-z^{k}\right)$. Let $\mathcal{L}$ be the sub- $\mathbb{Z}[q]$-lattice of $U^{+}$generated by $B_{\mathbf{i}}$, and let $\mathcal{L}^{*}$ be the sub-ZZZ $[q]$-lattice of $U^{+}$generated by $B_{\mathbf{i}}^{*}=\left\{E_{\mathbf{i}}(\mathbf{m})^{*}: \mathbf{m} \in \mathbb{Z}_{>0}^{N}\right\}$. Lusztig has shown that both $\mathcal{L}$ and $\mathcal{L}^{*}$ are independent of the choice of reduced decomposition i. Let $\eta$ be the $\mathbb{Q}$-algebra automorphism of $U$ fixing the generators $E_{i}$ and $F_{i}$, with $\eta\left(K_{\lambda}\right)=K_{-\lambda}$ and $\eta(q)=q^{-1}$. Lusztig [13] also shows:

Theorem 2.1. (Lusztig) Fix a reduced decomposition $\mathbf{i}$ of $w_{0}$. Then, for each $\mathbf{m} \in$ $\mathbb{Z}_{\geq 0}^{N}$, there is a unique element $B(\mathbf{m})=B_{\mathbf{i}}(\mathbf{m}) \in U^{+}$such that $\eta(B(\mathbf{m}))=B(\mathbf{m})$ and $B(\mathbf{m}) \in E(\mathbf{m})+q \mathcal{L}$. The set $\mathcal{B}=\left\{B(\mathbf{m}): \mathbf{m} \in \mathbb{Z}_{\geq 0}\right\}$ is a basis (called the canonical basis) of $U^{+}$which does not depend on $\mathbf{i}$. 
We'll call the parametrization $\mathbb{Z}_{\geq 0}^{N} \rightarrow \mathcal{B}, \mathbf{m} \mapsto B(\mathbf{m})$, Lusztig's parametrization of $\mathcal{B}$ (arising from $\mathbf{i}$ ). If $b \in B$, we denote by $L_{\mathbf{i}}(b)$ its Lusztig parameter (so $L_{\mathbf{i}}$ is the inverse of $\left.B_{\mathbf{i}}\right)$. For any $\mathbf{i}, \mathbf{i}^{\prime} \in R\left(w_{0}\right)$, we also have Lusztig's piecewise-linear reparametrization function $R_{\mathbf{i}}^{\mathbf{i}^{\prime}}=L_{\mathbf{i}^{\prime}} L_{\mathbf{i}}^{-1}$ (which can be regarded as a function from $\mathbb{R}^{N} \rightarrow \mathbb{R}^{N}$ via the same formula in terms of coordinates). The canonical basis was discovered independently by Kashiwara [8], who called it the global crystal basis. Let $\mathcal{B}^{*}=\left\{b^{*}: b \in \mathcal{B}\right\}$ denote the dual canonical basis of $U^{+}$. For $b \in \mathcal{B}$ we define $L_{\mathbf{i}}\left(b^{*}\right)$ to be $L_{\mathbf{i}}(b)$. Let $\sigma$ be the antihomomorphism of $U$ (as $\mathbb{Q}(q)$-algebra) taking $E_{i}$ to $E_{i}, F_{i}$ to $F_{i}$ and $K_{\lambda}$ to $K_{-\lambda}$. As in [11, Proposition 16] we have:

Proposition 2.2. Fix a reduced decomposition $\mathbf{i}$ of $w_{0}$. Then, for each $\mathbf{m} \in \mathbb{Z}_{\geq 0}^{N}$, the element $B(\mathbf{m})^{*}$ is the unique element $X$ of $U^{+}$with weight $\sum_{i} m_{i} \alpha_{i}$ such that

$$
\eta(X)=(-1)^{\operatorname{tr}(X)} q^{-\langle w t(X), w t(X)\rangle / 2} q_{X}^{-1} \sigma(X), X \in E(\mathbf{m})^{*}+q \mathcal{L}^{*},
$$

where $q_{X}=\prod_{i} q^{m_{i}}, w t(X)=\sum_{i} m_{i} \alpha_{i}$.

Fix a reduced decomposition $\mathbf{i}$ in $R\left(w_{0}\right)$. Let $\mathbf{e}_{k}, 1 \leq k \leq N$, be the canonical generators of $\mathbb{Z}_{\geq 0}^{N}$. We can define an ordering on the semigroup $\mathbb{Z}_{\geq 0}^{N}$ associated to $\mathbf{i}$ in the following way. The ordering $\prec_{\mathbf{i}}$ is generated by $\mathbf{m} \leq \mathbf{e}_{k}+\mathbf{e}_{k^{\prime}}, 1 \leq k<k^{\prime} \leq$ $N \Leftrightarrow E_{\mathbf{i}}(\mathbf{m})$ is a term of the PBW decomposition of $E_{\beta_{k}} E_{\beta_{k^{\prime}}}-q^{\left(\beta_{k^{\prime}}, \beta_{k}\right)} E_{\beta_{k^{\prime}}} E_{\beta_{k}}$. It will be denoted by $\prec$ if no confusion occurs.

Proposition 2.3. Fix i in $R\left(w_{0}\right)$. Then, for all $\mathbf{m}, \mathbf{n}$ in $\mathbb{Z}_{\geq 0}^{N}$ :

(i) if $\mathbf{m} \prec_{\mathbf{i}} \mathbf{n}$ then $\mathbf{m}$ is lower than $\mathbf{n}$ for the lexicographical ordering,

(ii) $B_{\mathbf{i}}(\mathbf{m})=E_{\mathbf{i}}(\mathbf{m})+\sum_{\mathbf{m} \prec \mathbf{n}} d_{\mathbf{m}}^{\mathbf{n}} E_{\mathbf{i}}(\mathbf{n}), d_{\mathbf{m}}^{\mathbf{n}} \in q \mathbb{Z}[q]$,

(iii) $B_{\mathbf{i}}(\mathbf{m})^{*}=E_{\mathbf{i}}(\mathbf{m})^{*}+q \sum_{\mathbf{n} \prec \mathbf{m}} c_{\mathbf{m}}^{\mathbf{n}} E_{\mathbf{i}}(\mathbf{n})^{*}, c_{\mathbf{m}}^{\mathbf{n}} \in \mathbb{Z}[q]$.

Proof: See [5, 2.1].

Let $q^{\mathbb{Z}} \mathcal{B}^{*}$ denote the set $\left\{q^{n} b^{*}: b \in \mathcal{B}, n \in \mathbb{Z}\right\}$. We therefore have:

Corollary 2.4. Let $\alpha \in Q^{+}$, and suppose $u \in U_{\alpha}^{+}$. Fix $\mathbf{i} \in R\left(w_{0}\right)$. Then $u \in q^{\mathbb{Z}} \mathcal{B}^{*}$ if and only if

(i) There is $f \in \mathbb{Z}\left[q, q^{-1}\right]$ such that $\sigma \eta(u)=f u$, and

(ii) $u=q^{k}\left(E(\mathbf{m})^{*}+q \sum_{\mathbf{n}<\mathbf{m}} c_{\mathbf{m}}^{\mathbf{n}} E_{\mathbf{i}}(\mathbf{n})^{*}\right)$, where $k \in \mathbb{Z}, c_{\mathbf{m}}^{\mathbf{n}} \in \mathbb{Z}[q]$ and $<$ denotes the lexicographic ordering.

Proof: It is immediate from Proposition 2.2 that if $u \in q^{\mathbb{Z}} \mathcal{B}^{*}$ then it satisfies (i) and (ii). If $k=0$ in part (ii), this ensures that, in the expansion of $u$ in terms of the dual canonical basis, $B_{\mathbf{i}}(\mathbf{m})^{*}$ occurs with coefficient 1 (see Proposition [2.3). It follows that the eigenvalue $f$ in part (i) must be the same as that appearing in (2.1), and it follows from Proposition 2.2 that $u \in \mathcal{B}^{*}$. The result for arbitrary $k$ follows.

Remark 2.5. We note that, from the proof, we can see that $u \in \mathcal{B}^{*}$ if and only if $u$ satisfies (i) and (ii) with $k=0$.

Two elements of the dual canonical basis $\mathcal{B}^{*}$ are said to be multiplicative if their product also lies in the dual canonical basis up to a power of $q$. They are said to $q$-commute if they commute up to a power of $q$. The following Corollary of Proposition 2.2 is due to Reineke [17, 4.5].

Corollary 2.6. If two elements of the dual canonical basis are multiplicative then they q-commute. 
Definition 2.7. There is a natural action of $U^{+}$on itself [1], in which each generator $E_{i}$ of $U^{+}$acts on $U^{+}$as a q-differential operator $\delta_{i}$. This is the unique action of $U^{+}$on itself satisfying the following properties:

(a) (Homogeneity) If $E \in U_{\alpha}^{+}, x \in U_{\gamma}^{+}$, then $E(x) \in U_{\gamma-\alpha}^{+}$.

(b) (Leibnitz formula)

$$
\delta_{i}(x y)=\delta_{i}(x) y+q^{-\left(\gamma, \alpha_{i}\right)} x \delta_{i}(y), \text { for } x \in U_{\gamma}^{+}, y \in U^{+} .
$$

(c) (Normalization) $\delta_{i}\left(E_{j}\right)=\left(1-q^{2}\right)^{-1} \delta_{i j}$ for $i, j=1,2, \ldots, n$.

We remark that formula (b) implies that for all $i$ and for $r \in \mathbb{Z}_{\geq 0}$, we have $\delta_{i}\left(E_{i}^{(r)}\right)=q^{-r+1}\left(1-q^{2}\right)^{-1} E_{i}^{(r-1)}$, which is easily checked by induction on $r\left(E_{i}^{(0)}\right.$ is interpreted as 1 and $E_{i}^{(-1)}$ as zero). For $u \in U^{+}$, set $\varphi_{i}(u)=\max \left\{r, \delta_{i}^{r}(u) \neq\right.$ $0\}$, and for $r \in \mathbb{Z}_{\geq 0}$, let $\delta_{i}^{(r)}$ denote the divided power $\frac{\delta_{i}^{r}}{r ! !}$. Define $\delta_{i}^{(\max )}(u):=$ $\delta_{i}^{\left(\varphi_{i}\left(b^{*}\right)\right)}(u)$. It is known that for $b \in \mathcal{B}, \delta_{i}^{(\max )}\left(b^{*}\right) \in \mathcal{B}^{*}$ (see [1, $\left.\S 1\right]$ ). If $\mathbf{i} \in R\left(w_{0}\right)$, set $a_{1}=\varphi_{i_{1}}\left(b^{*}\right), a_{2}=\varphi_{i_{2}}\left(\delta_{i_{1}}^{\left(a_{1}\right)}\left(b^{*}\right)\right), \ldots, a_{N}=\varphi_{i_{N}}\left(\delta_{i_{N-1}}^{\left(a_{N-1}\right)} \cdots \delta_{i_{1}}^{\left(a_{1}\right)}\left(b^{*}\right)\right)$; then $\left(a_{1}, a_{2}, \ldots, a_{N}\right)$ is known as the string of $b\left(\right.$ or $\left.b^{*}\right)$ in direction $\mathbf{i}$ [1]; this coincides with the string of $b$ arising from Kashiwara's approach to $\mathcal{B}$ (see $[9$ and the end of Section 2 in $[16$ ).

\section{PBW-STRINGS}

In this section we will show how the Lusztig parametrization of a dual canonical basis element can also be regarded as a string (in a similar sense to the above). We define new operators $\Delta_{i}, i=1,2, \ldots, n$, depending on a choice of reduced decomposition for $w_{0}$, which play the role of the operators $\delta_{i}^{(\max )}$ for the PBW parametrization. We first of all note that $\delta_{i}^{(\max )}$ is a well-defined operator on all of $U^{+}$.

Definition 3.1. Let $\widetilde{w}=s_{i_{1}} s_{i_{2}} \cdots s_{i_{t}}$ be a reduced decomposition for $w \in W$. Let $\Delta_{1}=\delta_{i_{1}}^{(\max )}, \Delta_{2}=T_{i_{1}} \delta_{i_{2}}^{(\max )} T_{i_{1}}^{-1}, \ldots, \Delta_{t}=\left(T_{i_{1}} \cdots T_{i_{t-1}}\right) \delta_{i_{t}}^{(\max )}\left(T_{i_{1}} \cdots T_{i_{t-1}}\right)^{-1}$, operators on $U^{+}$(with codomain $\left.U\right)$.

We will use the following:

Lemma 3.2. (Saito) Let $i \in\{1,2, \ldots, n\}$. Then $T_{i}\left(U^{+}\right) \cap U^{+}=\operatorname{ker} \delta_{i}$.

Proof: See [19].

Lemma 3.3. Let $\mathbf{i} \in R\left(w_{0}\right)$, and $\mathbf{m}=\left(m_{1}, m_{2}, \ldots, m_{N}\right) \in \mathbb{Z}_{\geq 0}^{N}$. Then

$$
\delta_{i_{1}}^{(\max )}\left(E_{\mathbf{i}}(\mathbf{m})^{*}\right)=E_{\mathbf{i}}\left(0, m_{2}, m_{3}, \ldots, m_{N}\right)^{*} .
$$

Proof: Let $\gamma_{\mathbf{m}}=\prod_{t=1}^{N} \psi_{m_{t}}\left(q^{2}\right)$, so that $E_{\mathbf{i}}(\mathbf{m})^{*}=\gamma_{\mathbf{m}} E_{\mathbf{i}}(\mathbf{m})$. Then $E_{\mathbf{i}}^{\mathbf{m}}=$ $E_{i_{1}}^{\left(m_{1}\right)} T_{i_{1}}(y)$, where $y=E_{i_{2}}^{\left(m_{2}\right)} T_{i_{2}}\left(E_{i_{3}}^{\left(m_{3}\right)}\right) \cdots T_{i_{2}} T_{i_{3}} \cdots T_{i_{N-1}}\left(E_{i_{N}}^{\left(m_{N}\right)}\right) \in U^{+}$. Using Lemma 3.2 the formula for $\gamma_{\mathbf{m}}$ and the fact that $\delta_{i_{1}}\left(E_{i_{1}}^{(m)}\right)=q^{-m+1}(1-$ $\left.q^{2}\right)^{-1} E_{i_{1}}^{(m-1)}$ the result follows.

We can now prove a Lemma giving basic properties of the Lusztig parametrization with respect to the operators $\Delta_{i}$. 
Lemma 3.4. Let $b \in \mathcal{B}$, and suppose $\mathbf{i} \in R\left(w_{0}\right)$ and $\Delta_{1}, \Delta_{2}, \ldots, \Delta_{N}$ are as above. Let $L_{\mathbf{i}}(b)=\left(m_{1}, m_{2}, \ldots, m_{N}\right) \in \mathbb{Z}_{\geq 0}^{N}$. Then, for $k=1,2, \ldots, N$, we have:

(i) $\Delta_{k} \Delta_{k-1} \cdots \Delta_{1}\left(b^{*}\right) \in \mathcal{B}^{*}$,

(ii) $\varphi_{i_{k}}\left(\left(T_{i_{1}} \cdots T_{i_{k-1}}\right)^{-1} \Delta_{k-1} \cdots \Delta_{1}\left(b^{*}\right)\right)=m_{k}$,

(iii) $L_{\mathbf{i}}\left(\Delta_{k} \Delta_{k-1} \cdots \Delta_{1}\left(b^{*}\right)\right)=\left(0,0, \ldots, 0, m_{k+1}, m_{k+2}, \ldots, m_{N}\right)$.

Proof: Since $\delta_{i_{1}}^{(\max )}$ preserves $\mathcal{B}^{*}$, we see that $\Delta_{1}\left(b^{*}\right)$ lies in $\mathcal{B}^{*}$, so (i) holds for $k=1$, but we need more precise information. Since $b^{*} \in \mathcal{B}^{*}$, we know by [5] (see also Proposition 2.3) that

$$
b^{*}=E_{\mathbf{i}}(\mathbf{m})^{*}+q x_{0},
$$

where $x_{0}$ is a $\mathbb{Z}[q]$-linear combination of dual PBW-basis elements $E_{\mathbf{i}}(\mathbf{n})^{*}$ with $\mathbf{n}<\mathbf{m}$ (and in particular, satisfying $n_{1} \leq m_{1}$ ). Here $<$ denotes the lexicographic ordering. By Lemma 3.3 we see that

$$
\delta_{i_{1}}^{(\max )}\left(b^{*}\right)=E_{\mathbf{i}}\left(0, m_{2}, m_{3}, \ldots, m_{N}\right)^{*}+q x_{1},
$$

where $x_{1}$ is a $\mathbb{Z}[q]$-linear combination of elements of the form $E_{\mathbf{i}}(\mathbf{n})^{*}$ with $\mathbf{n}=$ $\left(0, n_{2}, n_{3}, \ldots, n_{N}\right)<\mathbf{m}$ for all $\mathbf{n}$ occurring in the sum. By Corollary 2.4 we have that $L_{\mathbf{i}}\left(\Delta_{1}\left(b^{*}\right)\right)=\left(0, m_{2}, m_{3}, \ldots, m_{N}\right)$, so (ii) holds for $k=1$. It is also clear that $\varphi_{i_{1}}\left(b^{*}\right)=m_{1}$, so (iii) holds for $k=1$. We thus see that (i), (ii) and (iii) all hold for $k=1$.

¿From the above form for $\Delta_{1}\left(b^{*}\right)$, we have that

$$
T_{i_{1}}^{-1}\left(\Delta_{1}\left(b^{*}\right)\right)=E_{\mathbf{i}^{\prime}}\left(m_{2}, m_{3}, \ldots, m_{N}, 0\right)^{*}+q y_{1},
$$

where $\mathbf{i}^{\prime}=s_{i_{2}} s_{i_{3}} \cdots s_{i_{N}} s_{i_{1}^{*}}, i_{1}^{*}$ is the index of the simple root $-w\left(\alpha_{i_{1}}\right)$ (the Chevalley automorphism applied to $i_{1}$ ), and $y_{1}$ is a $\mathbb{Z}[q]$-linear combination of dual PBW-basis elements $E_{\mathbf{i}^{\prime}}(\mathbf{n})^{*}$ with $\mathbf{n}=\left(n_{2}, n_{3}, \ldots, n_{N}, 0\right)<\left(m_{2}, m_{3}, \ldots, m_{N}, 0\right)$ and therefore satisfies Corollary 2.4(ii) (with $k=0$ ). Since for all $i, T_{i}^{-1} \sigma \eta$ and $\sigma \eta T_{i}^{-1}$ differ on weight spaces by plus or minus a power of $q$, it follows that $T_{i_{1}}^{-1}\left(\Delta_{1}\left(b^{*}\right)\right)$ satisfies Corollary 2.4(i), and therefore (see the remark after Corollary 2.4(i)) lies in $\mathcal{B}^{*}$. Since $\delta_{i_{2}}^{(\max )}$ preserves $\mathcal{B}^{*}$, we thus obtain that $\delta_{i_{2}}^{(\max )} T_{i_{1}}^{-1} \Delta_{1}\left(b^{*}\right) \in \mathcal{B}^{*}$. Arguing as above, with $i_{2}$ playing the role of $i_{1}$, we obtain that

$$
\delta_{i_{2}}^{(\max )} T_{i_{1}}^{-1} \Delta_{1}\left(b^{*}\right)=E_{\mathbf{i}^{\prime}}\left(0, m_{3}, m_{4}, \ldots, m_{N}, 0\right)^{*}+q y_{2},
$$

where $y_{2}$ is a $\mathbb{Z}[q]$-linear combination of dual PBW-basis elements of the form $E_{\mathbf{i}^{\prime}}(\mathbf{n})^{*}$ with $\mathbf{n}=\left(0, n_{3}, n_{4}, \ldots, n_{N}, 0\right)<\left(0, m_{3}, m_{4}, \ldots, m_{N}, 0\right)$. We also obtain that $\varphi_{i_{2}}\left(T_{i_{1}}^{-1} \Delta_{1}\left(b^{*}\right)\right)=m_{2}$ (part (ii) for $k=2$ ). Applying $T_{i_{1}}$ we obtain that

$$
\Delta_{2} \Delta_{1}\left(b^{*}\right)=T_{i_{1}} \delta_{i_{2}}^{(\max )} T_{i_{1}}^{-1} \Delta_{1}\left(b^{*}\right)=E_{\mathbf{i}}\left(0,0, m_{3}, m_{4}, \ldots, m_{N}\right)^{*}+q x_{2},
$$

where $x_{2}$ is a $\mathbb{Z}[q]$-linear combination of dual PBW-basis elements of the form $E_{\mathbf{i}}(\mathbf{n})^{*}$ with $\mathbf{n}=\left(0,0, n_{3}, n_{4}, \ldots, n_{N}\right)<\left(0,0, m_{3}, m_{4}, \ldots, m_{N}\right)$. Arguing as above for $T_{i_{1}}^{-1}$ and applying Corollary 2.4 we obtain that $\Delta_{2} \Delta_{1}\left(b^{*}\right) \in \mathcal{B}^{*}$ and that $L_{\mathbf{i}}\left(\Delta_{2} \Delta_{1}\left(b^{*}\right)\right)=\left(0,0, m_{3}, m_{4}, \ldots, m_{N}\right)$. We thus see that (i) and (iii) hold for $k=2$. It is now clear that an inductive argument gives (i),(ii) and (iii) for $k=1,2, \ldots, N$.

Given a reduced decomposition $\widetilde{w}=s_{i_{1}} s_{i_{2}} \cdots s_{i_{t}}$ for an element $w \in W$, we define the PBW-string $L_{\widetilde{w}}\left(b^{*}\right)$ of a dual canonical basis element $b^{*}$ in direction $\widetilde{w}$ as follows. Let $\mathbf{i}=s_{i_{1}} s_{i_{2}} \cdots s_{i_{t}} s_{i_{t+1}} \cdots s_{i_{N}}$ be any completion of $\widetilde{w}$ to a reduced decomposition for $w_{0}$. Then let $L_{\widetilde{w}}\left(b^{*}\right)=\left(m_{1}, m_{2}, \ldots, m_{t}\right)$ where $L_{\mathbf{i}}\left(b^{*}\right)=\left(m_{1}, m_{2}, \ldots, m_{N}\right)$. It is clear from Lemma 3.4 that this is well-defined. 
We make definitions for PBW-strings in the same way as Berenstein and Zelevinsky [1] for usual strings.

Definition 3.5. Let $\mathbf{i} \in R\left(w_{0}\right)$. A PBW $\mathbf{i}$-wall is defined to be a hyperplane in $\mathbb{R}^{N}$ given by the equation $a_{k}=a_{k+2}$ for some index $k$ such that $i_{k}=i_{k+2}=i_{k+1} \pm 1$. Let $\mathbf{m} \in \mathbb{R}_{>0}^{N}$. We say that $\mathbf{m}$ is $P B W \mathbf{i}$-regular if for every $\mathbf{i}^{\prime} \in R\left(w_{0}\right)$ the point $R_{\mathbf{i}}^{\mathbf{i}^{\prime}}(\mathbf{m})$ does not lie on any $P B W \mathbf{i}^{\prime}$-wall. We define the $P B W \mathbf{i}$-linearity domains to be the closures of the connected components of the set of PBW $\mathbf{i}$-regular points.

As in the string case [1, 2.8], we have the following justification for the terminology; the proof is basically the same, as $R_{\mathbf{i}}^{\mathbf{i}^{\prime}}$ is very similar to the string reparametrization function.

Proposition 3.6. Every $P B W$ i-linearity domain is a polyhedral convex cone in $\mathbb{R}_{\geq 0}^{N}$. Two points $\mathbf{m}, \mathbf{m}^{\prime}$ in $\mathbb{R}_{\geq 0}^{N}$ lie in a single $P B W \mathbf{i}$-linearity domain if and only if $^{-0}$

for every $\mathbf{i}^{\prime} \in R\left(w_{0}\right)$.

$$
R_{\mathbf{i}}^{\mathbf{i}^{\prime}}\left(\mathbf{m}+\mathbf{m}^{\prime}\right)=R_{\mathbf{i}}^{\mathbf{i}^{\prime}}(\mathbf{m})+R_{\mathbf{i}}^{\mathbf{i}^{\prime}}\left(\mathbf{m}^{\prime}\right)
$$

We can now prove the analogue of [1, 2.9]:

Theorem 3.7. Let $b^{*}, b^{*}$ be elements of the dual canonical basis that q-commute. Then, for every $\mathbf{i} \in R\left(w_{0}\right)$, the PBW strings $\mathbf{m}=L_{\mathbf{i}}\left(b^{*}\right)$ and $\mathbf{m}^{\prime}=L_{\mathbf{i}}\left(b^{\prime *}\right)$ belong to a single $\mathbf{i}$-linearity domain.

Proof: We follow the proof of Berenstein and Zelevinsky [1; however there will be some differences, so we include the details. We know by Proposition 3.6 that it is enough to show that, for every $\mathbf{i}^{\prime} \in R\left(w_{0}\right), R_{\mathbf{i}}^{\mathbf{i}^{\prime}}\left(\mathbf{m}+\mathbf{m}^{\prime}\right)=R_{\mathbf{i}}^{\mathbf{i}^{\prime}}(\mathbf{m})+R_{\mathbf{i}}^{\mathbf{i}^{\prime}}\left(\mathbf{m}^{\prime}\right)$. Thus, it is enough to show that $\mathbf{m}$ and $\mathbf{m}^{\prime}$ are not separated by any PBW $\mathbf{i}$-wall. Suppose that a $\mathbf{i}$-wall corresponds to the move

$$
\mathbf{i}=s_{i_{1}} \cdots s_{i_{t}} s_{i} s_{j} s_{i} s_{i_{t+4}} \cdots s_{i_{N}} \mapsto s_{i_{1}} \cdots s_{i_{t}} s_{j} s_{i} s_{j} s_{i_{t+4}} \cdots s_{i_{N}}=\mathbf{i}^{\prime} .
$$

By [1,3.6] and the fact that the $T_{i}$ are algebra automorphisms, the elements $\left(T_{i_{k}} T_{i_{k-1}} \cdots T_{i_{1}}\right)^{-1} \Delta_{t} \Delta_{t-1} \cdots \Delta_{1}\left(b^{*}\right)$ and $\left(T_{i_{k}} T_{i_{k-1}} \cdots T_{i_{1}}\right)^{-1} \Delta_{t} \Delta_{t-1} \cdots \Delta_{1}\left(b^{\prime *}\right)$ $q$-commute. Replacing $b^{*}$ and $b^{* *}$ by $\left(T_{i_{k}} T_{i_{k-1}} \cdots T_{i_{1}}\right)^{-1} \Delta_{t} \Delta_{t-1} \cdots \Delta_{1}\left(b^{*}\right)$ and $\left(T_{i_{k}} T_{i_{k-1}} \cdots T_{i_{1}}\right)^{-1} \Delta_{t} \Delta_{t-1} \cdots \Delta_{1}\left(b^{*}\right)$, we can assume that $t=0$ (using Lemma 3.4). Let $L_{i j i}=L_{s_{i} s_{j} s_{i}}\left(b^{*}\right)=\left(m_{1}, m_{2}, m_{3}\right)$ and $L_{i j i}^{\prime}=L_{s_{i} s_{j} s_{i}}\left(b^{\prime *}\right)=\left(m_{1}^{\prime}, m_{2}^{\prime}, m_{3}^{\prime}\right)$ be the PBW-strings of $b^{*}$ and $b^{*}$ in direction $s_{i} s_{j} s_{i}$. It is sufficient to show that the integers $m_{1}-m_{3}$ and $m_{1}^{\prime}-m_{3}^{\prime}$ are of the same sign. Now let $\Delta_{1}, \Delta_{2}, \Delta_{3}$ be the operators on $\mathcal{B}^{*}$ associated to the reduced decomposition $s_{i} s_{j} s_{i}$ as in Definition 3.1 Let $b_{0}^{*}=\Delta_{3} \Delta_{2} \Delta_{1}\left(b^{*}\right)$, and let $b_{0}^{\prime *}=\Delta_{3} \Delta_{2} \Delta_{1}\left(b^{* *}\right)$. Suppose that $b^{\prime *} b^{*}=q^{n} b^{*} b^{\prime *}$ and $b_{0}^{* *} b_{0}^{*}=q^{n_{0}} b_{0}^{*} b_{0}^{* *}$ for integers $n, n_{0}$. Then we have

$$
\begin{aligned}
\Delta_{3} \Delta_{2} \Delta_{1}\left(b^{*} b^{\prime *}\right) & =T_{i} T_{j} \delta_{i}^{(\max )} T_{j}^{-1} T_{i}^{-1} \delta_{j}^{(\max )} T_{i}^{-1} \delta_{i}^{(\max )}\left(b b^{\prime}\right) \\
& =q^{r_{1}} \Delta_{3} \Delta_{2} \Delta_{1}\left(b^{*}\right) \Delta_{3} \Delta_{2} \Delta_{1}\left(b^{\prime *}\right) \\
& =q^{r_{1}} b_{0}^{*} b_{0}^{\prime *}
\end{aligned}
$$

where

$$
r_{1}=\Phi_{i, \gamma}\left(m_{1}, m_{1}^{\prime}\right)+\Phi_{j, s_{i}\left(\gamma-m_{1} \alpha_{i}\right)}\left(m_{2}, m_{2}^{\prime}\right)+\Phi_{i, s_{j} s_{i}\left(\gamma-m_{1} \alpha_{i}\right)-m_{2} \alpha_{j}},
$$

and $\gamma$ is the degree of $b^{*}$. Here $\Phi_{k, \mu}(n, m)=n m-\left(\mu, m \alpha_{k}\right)=m\left(n-\left(\mu, \alpha_{k}\right)\right)$ is defined as in [1 Proposition 3.1] and we are using [1 (3.6)]. We also use the 
fact that for all $\alpha \in Q^{+}, T_{i}^{ \pm 1}\left(U_{\alpha}^{+}\right) \cap U^{+} \subset U_{s_{i}(\alpha)}^{+}$. Expanding and simplifying, we obtain

$$
\begin{aligned}
r_{1}= & m_{1} m_{1}^{\prime}+m_{2} m_{2}^{\prime}+m_{3} m_{3}^{\prime}+m_{1} m_{2}^{\prime}+m_{2} m_{3}^{\prime}-m_{1} m_{3}^{\prime}- \\
& m_{1}^{\prime}\left(\gamma, \alpha_{i}\right)-m_{2}^{\prime}\left(\gamma, \alpha_{i}+\alpha_{j}\right)-m_{3}\left(\gamma, \alpha_{j}\right) .
\end{aligned}
$$

Similarly, we obtain that $\Delta_{3} \Delta_{2} \Delta_{1}\left(b^{*} b^{\prime *}\right)=q^{r_{2}} \Delta_{3} \Delta_{2} \Delta_{1}\left(b^{*}\right) \Delta_{3} \Delta_{2} \Delta_{1}\left(b^{* *}\right)$, where

$$
\begin{aligned}
r_{2}= & m_{1}^{\prime} m_{1}+m_{2}^{\prime} m_{2}+m_{3}^{\prime} m_{3}+m_{1}^{\prime} m_{2}+m_{2}^{\prime} m_{3}-m_{1}^{\prime} m_{3}- \\
& m_{1}\left(\gamma^{\prime}, \alpha_{i}\right)-m_{2}\left(\gamma^{\prime}, \alpha_{i}+\alpha_{j}\right)-m_{3}\left(\gamma^{\prime}, \alpha_{j}\right),
\end{aligned}
$$

and $\gamma^{\prime}$ is the weight of $b^{\prime *}$. We thus have:

$$
n_{0}-n=r_{1}-r_{2} .
$$

Let $L_{j i j}=L_{s_{j} s_{i} s_{j}}\left(b^{*}\right)=\left(n_{1}, n_{2}, n_{3}\right)$ and $L_{j i j}^{\prime}=L_{s_{j} s_{i} s_{j}}\left(b^{*}\right)=\left(n_{1}^{\prime}, n_{2}^{\prime}, n_{3}^{\prime}\right)$ be the PBW-strings of $b^{*}$ and $b^{* *}$ in direction $s_{j} s_{i} s_{j}$. Then we know that $R\left(m_{1}, m_{2}, m_{3}\right)=$ $\left(n_{1}, n_{2}, n_{3}\right)$ and that $R\left(m_{1}^{\prime}, m_{2}^{\prime}, m_{3}^{\prime}\right)=\left(n_{1}^{\prime}, n_{2}^{\prime}, n_{3}^{\prime}\right)$, where $R=R_{s_{1} s_{2} s_{1}}^{s_{2} s_{1} s_{2}}$ is Lusztig's piecewise reparametrization function associated to the canonical basis in type $A_{2}$ (see 13]). Let $\Delta_{1}^{\prime}, \Delta_{2}^{\prime}, \Delta_{3}^{\prime}$ be the operators on $\mathcal{B}^{*}$ associated to the reduced decomposition $s_{j} s_{i} s_{j}$ as in Definition 3.1 It follows from Lemma 3.4 that $b_{0}^{*}=$ $\Delta_{3}^{\prime} \Delta_{2}^{\prime} \Delta_{1}^{\prime}\left(b^{*}\right)$, and that $b_{0}^{\prime *}=\Delta_{3}^{\prime} \Delta_{2}^{\prime} \Delta_{1}^{\prime}\left(b^{\prime *}\right)$. Hence, a similar argument to that given above shows that

$$
n_{0}-n=r_{1}^{\prime}-r_{2}^{\prime}
$$

where

$$
\begin{aligned}
r_{1}^{\prime}= & n_{1} n_{1}^{\prime}+n_{2} n_{2}^{\prime}+n_{3} n_{3}^{\prime}+n_{1} n_{2}^{\prime}+n_{2} n_{3}^{\prime}-n_{1} n_{3}^{\prime}- \\
& n_{1}^{\prime}\left(\gamma, \alpha_{i}\right)-n_{2}^{\prime}\left(\gamma, \alpha_{i}+\alpha_{j}\right)-n_{3}\left(\gamma, \alpha_{j}\right)
\end{aligned}
$$

and

$$
\begin{aligned}
r_{2}^{\prime}= & n_{1}^{\prime} n_{1}+n_{2}^{\prime} n_{2}+n_{3}^{\prime} n_{3}+n_{1}^{\prime} n_{2}+n_{2}^{\prime} n_{3}-n_{1}^{\prime} n_{3}- \\
& n_{1}\left(\gamma^{\prime}, \alpha_{i}\right)-n_{2}\left(\gamma^{\prime}, \alpha_{i}+\alpha_{j}\right)-n_{3}\left(\gamma^{\prime}, \alpha_{j}\right) .
\end{aligned}
$$

Suppose now that $m_{1}>m_{3}$ and $m_{1}^{\prime}<m_{3}^{\prime}$. Then $\left(n_{1}, n_{2}, n_{3}\right)=R\left(m_{1}, m_{2}, m_{3}\right)=$ $\left(m_{2}, m_{3}, m_{1}+m_{2}-m_{3}\right)$ and $\left(n_{1}^{\prime}, n_{2}^{\prime}, n_{3}^{\prime}\right)=R\left(m_{1}^{\prime}, m_{2}^{\prime}, m_{3}^{\prime}\right)=\left(m_{2}^{\prime}+m_{3}^{\prime}-m_{1}^{\prime}, m_{1}^{\prime}, m_{2}^{\prime}\right)$. Equating 3.1 and 3.2 we obtain: $\left(m_{1}-m_{3}\right)\left(m_{3}^{\prime}-m_{1}^{\prime}\right)=0$, which is a contradiction, as each of $m_{1}-m_{3}$ and $m_{3}^{\prime}-m_{1}^{\prime}$ has been assumed to be positive. A similar argument shows that we cannot have $m_{1}<m_{3}$ and $m_{1}^{\prime}>m_{3}^{\prime}$. It follows that $\mathbf{m}$ and $\mathbf{m}^{\prime}$ cannot be separated by a PBW $\mathbf{i}$-wall, and the Theorem is proved.

\section{Properties of PBW i-Linearity domains}

In this section we show that the set of i-linearity domains forms a fan. We start with a key proposition (which we shall also need in section 5 when we discuss $q$-commuting properties of the dual canonical basis). We call the connected components of the set of PBW i-regular points PBW i-chambers, so that PBW i-linearity domains are the closures of PBW i-chambers (see [1 §8]).

Lemma 4.1. Let $\mathbf{m}, \mathbf{m}^{\prime} \in \mathbb{R}_{\geq 0}^{N}$. Then $\mathbf{m}$ and $\mathbf{m}^{\prime}$ lie in a single $P B W \mathbf{i}$-linearity domain if and only if for all $\mathbf{i}^{\prime} \in R\left(w_{0}\right), R_{\mathbf{i}}^{\mathbf{i}^{\prime}}(\mathbf{m})$ and $R_{\mathbf{i}}^{\mathbf{i}^{\prime}}\left(\mathbf{m}^{\prime}\right)$ are weakly on the same side of all $P B W \mathbf{i}^{\prime}$-walls. 
Proof: By definition, $\mathbf{m}$ and $\mathbf{m}^{\prime}$ lie in a single PBW $\mathbf{i}$-chamber if and only if for all $\mathbf{i}^{\prime} \in R\left(w_{0}\right), R_{\mathbf{i}}^{\mathbf{i}^{\prime}}(\mathbf{m})$ and $R_{\mathbf{i}}^{\mathbf{i}^{\prime}}\left(\mathbf{m}^{\prime}\right)$ are strictly on the same side of all PBW $\mathbf{i}^{\prime}$-walls. Since PBW $\mathbf{i}$-linearity domains are the closures of PBW $\mathbf{i}$-chambers, it follows from the continuity of the functions $R_{\mathbf{i}}^{\mathbf{i}^{\prime}}$ that if $\mathbf{m}$ and $\mathbf{m}^{\prime}$ lie in a single PBW $\mathbf{i}$-linearity domain, then for all $\mathbf{i}^{\prime} \in R\left(w_{0}\right), R_{\mathbf{i}}^{\mathbf{i}^{\prime}}(\mathbf{m})$ and $R_{\mathbf{i}}^{\mathbf{i}^{\prime}}\left(\mathbf{m}^{\prime}\right)$ are weakly on the same side of all PBW i'-walls. Conversely, if for all $\mathbf{i}^{\prime} \in R\left(w_{0}\right), R_{\mathbf{i}}^{\mathbf{i}^{\prime}}(\mathbf{m})$ and $R_{\mathbf{i}}^{\mathbf{i}^{\prime}}\left(\mathbf{m}^{\prime}\right)$ are weakly on the same side of all PBW $\mathbf{i}^{\prime}$-walls, then for all $\mathbf{i}^{\prime} \in R\left(w_{0}\right)$,

$$
R_{\mathbf{i}}^{\mathbf{i}^{\prime}}\left(\mathbf{m}+\mathbf{m}^{\prime}\right)=R_{\mathbf{i}}^{\mathbf{i}^{\prime}}(\mathbf{m})+R_{\mathbf{i}}^{\mathbf{i}^{\prime}}\left(\mathbf{m}^{\prime}\right) \text {. }
$$

This can be proved by induction on the number of braid relations needed to take $\mathbf{i}$ to $\mathbf{i}^{\prime}$ (see the proof of [1 8.1]). By Proposition 3.6] this implies that $\mathbf{m}$ and $\mathbf{m}^{\prime}$ lie in a single $\mathrm{PBW}$ i-linearity domain.

Proposition 4.2. Let $\mathbf{i} \in R\left(w_{0}\right)$. Suppose that $\mathbf{m}_{\mathbf{i}}, 1 \leq i \leq k$ and $\sum_{i=1}^{k} \mathbf{m}_{i}$ all ie in a single $P B W \mathbf{i}$-linearity domain $X$. Suppose also that $\sum_{i=1}^{k} \mathbf{m}_{i}$ and $\mathbf{q}$ both lie in a single $P B W \mathbf{i}$-linearity domain (not necessarily the same as $X$ ). Then $\mathbf{m}_{\mathbf{i}}$, $i=1,2, \ldots, k, \sum_{i=1}^{k} \mathbf{m}_{i}$ and $\mathbf{q}$ all lie in a single $P B W \mathbf{i}$-linearity domain.

Proof: We use Lemma 4.1 throughout. By the assumptions in the Proposition, we have:

(i) $R_{\mathbf{i}}^{\mathbf{i}^{\prime}}\left(\mathbf{m}_{i}\right), 1 \leq i \leq k$ and $R_{\mathbf{i}}^{\mathbf{i}^{\prime}}\left(\sum_{i=1}^{k} \mathbf{m}_{i}\right)$ are weakly on the same side of all PBW $\mathbf{i}^{\prime}$-walls, and:

(ii) $R_{\mathbf{i}}^{\mathbf{i}^{\prime}}(\mathbf{q})$ and $R_{\mathbf{i}}^{\mathbf{i}^{\prime}}\left(\sum_{i=1}^{k} \mathbf{m}_{i}\right)$ are weakly on the same side of all PBW $\mathbf{i}^{\prime}$-walls. Let $H$ be a PBW $\mathbf{i}^{\prime}$-wall. Firstly, let $\mathbf{i}^{\prime} \in R\left(w_{0}\right)$ be such that $R_{\mathbf{i}}^{\mathbf{i}^{\prime}}\left(\sum_{i=1}^{k} \mathbf{m}_{i}\right)$ does not lie on $H$. Then $R_{\mathbf{i}}^{\mathbf{i}^{\prime}}\left(\mathbf{m}_{i}\right), i=1,2, \ldots, k, R_{\mathbf{i}}^{\mathbf{i}^{\prime}}\left(\sum_{i=1}^{k} \mathbf{m}_{i}\right)$ and $R_{\mathbf{i}}^{\mathbf{i}^{\prime}}(\mathbf{q})$ are all weakly on the same side of $H$. Next, suppose that $\mathbf{i}^{\prime} \in R\left(w_{0}\right)$ is such that $R_{\mathbf{i}}^{\mathbf{i}^{\prime}}\left(\sum_{i=1}^{k} \mathbf{m}_{i}\right)$ does lie on $H$. By (i) and Proposition [3.6] $R_{\mathbf{i}}^{\mathbf{i}^{\prime}}\left(\mathbf{m}_{i}\right)$ lies on $H$ for $1 \leq i \leq k$. Hence $R_{\mathbf{i}}^{\mathbf{i}^{\prime}}\left(\mathbf{m}_{i}\right), 1 \leq i \leq k, R_{\mathbf{i}}^{\mathbf{i}^{\prime}}\left(\sum_{i=1}^{k} \mathbf{m}_{i}\right)$ and $R_{\mathbf{i}}^{\mathbf{i}^{\prime}}(\mathbf{q})$ are all weakly on the same side of $H$. It follows (using Lemma 4.1 again) that the $\mathbf{m}_{\mathbf{i}}, i=1,2, \ldots, k, \sum_{i=1}^{k} \mathbf{m}_{i}$ and $\mathbf{q}$ all lie in a single PBW i-linearity domain.

Remark 4.3. If, in the assumptions and conclusion of the proposition, the property "lie in a single PBW $\mathbf{i}$-linearity domain" is replaced by the property " $q$-commute" (and the tuples involved are assumed to lie in $\mathbb{Z}_{\geq 0}^{N}$ ), the result is not clear.

Recall that a strongly convex polyhedral cone is a convex polyhedral cone $C$ for which $v \in C$ implies $-v \notin C$. A set of strongly convex polyhedral cones is said to form a fan if the face of every cone in the set lies in the set and if the intersection of any two cones in the set lies again in the set.

Corollary 4.4. Let $\mathbf{i} \in R\left(w_{0}\right)$. Then the set of PBW $\mathbf{i}$-linearity domains (together with all their faces) forms a fan in $\mathbb{R}^{N}$.

Proof: We first of all note that each PBW i-linearity domain is a convex polyhedral cone (by Proposition 3.6) and thus is in fact a strongly convex polyhedral cone as it contains no point with negative coordinates. The same is therefore true for all of its faces. We now show that if $C$ and $C^{\prime}$ are distinct PBW i-linearity domains, then $C \cap C^{\prime}$ is a face of $C$ and of $C^{\prime}$. Suppose that $C \cap C^{\prime}$ is not a face of $C$. Then $C \cap C^{\prime} \subsetneq F$, where $F$ is a face of $C$ (since $C$ and $C^{\prime}$ are convex polyhedral cones). Let $\left(C^{\prime}\right)^{\circ}$ denote the interior of $C^{\prime}$; we will use similar notation for the 
interiors of other cones. Fix $\mathbf{p} \in F \backslash C$. Then, we can choose $\mathbf{m} \in C \cap C^{\prime}$ such that $\mathbf{m}+\mathbf{p} \in C \cap C^{\prime}$, since $C \cap C^{\prime}$ and $F$ are convex polyhedral cones. Now, let $\mathbf{q}$ be in $\left(C^{\prime}\right)^{\circ}$. We have that $\mathbf{q}$ and $\mathbf{m}+\mathbf{p}$ lie in a single $\mathrm{PBW} \mathbf{i}$-linearity domain, and $\mathbf{m}, \mathbf{p}$ and $\mathbf{m}+\mathbf{p}$ lie in a single PBW $\mathbf{i}$-linearity domain. So by Proposition $4.2 \mathbf{q}$ and $\mathbf{p}$ both lie in a single PBW i-linearity domain. But, as $\mathbf{q} \in\left(C^{\prime}\right)^{\circ}, C^{\prime}$ is the only PBW i-linearity domain containing $\mathbf{q}$. Since $\mathbf{p} \notin C^{\prime}$, we have a contradiction, and we see that $C \cap C^{\prime}$ is a face of $C$ (and similarly, we can see that it is a face of $C^{\prime}$ ).

We next consider the case where $C, C^{\prime}$ are faces of distinct PBW-linearity domains, $P$ and $P^{\prime}$. We show that $C \cap C^{\prime}$ is a face of $C$ and of $C^{\prime}$. Suppose that $C \cap C^{\prime}$ is not a face of $C$. Then, as above, $C \cap C^{\prime} \subsetneq F$, where $F$ is a face of $C$. As above, we can then choose $\mathbf{q} \in\left(P^{\prime}\right)^{\circ}, \mathbf{p} \in F \backslash C^{\prime}$ and $\mathbf{m} \in C \cap C^{\prime}$ such that $\mathbf{m}+\mathbf{p} \in C \cap C^{\prime}$, since $C \cap C^{\prime}$ and $F$ are convex polyhedral cones. Note that the first two properties imply that $\mathbf{p} \notin P^{\prime}$. This is because $\mathbf{p}$ lies in the linear span of $C^{\prime} \cap C$, so $\mathbf{p}$ lies in the linear span of $C^{\prime}$. Since $P^{\prime}$ is convex, if $\mathbf{p}$ was in $P^{\prime}$ and in the linear span of its face $C^{\prime}$, it would have to lie in $C^{\prime}$, a contradiction to the assumption that $\mathbf{p} \in F \backslash C^{\prime}$. So $\mathbf{q}$ and $\mathbf{m}+\mathbf{p}$ lie in a single PBW $\mathbf{i}$-linearity domain $P^{\prime}$, while $\mathbf{m}, \mathbf{p}$ and $\mathbf{m}+\mathbf{p}$ all lie in a single PBW $\mathbf{i}$-linearity domain $P$. Proposition 4.2 asserts that $\mathbf{q}$ and $\mathbf{p}$ both lie in a single PBW $\mathbf{i}$-linearity domain, but as $\mathbf{q} \in\left(P^{\prime}\right)^{\circ}, P^{\prime}$ is the only PBW $\mathbf{i}$-linearity domain containing $\mathbf{q}$, while $\mathbf{p} \notin P^{\prime}$, so we have a contradiction. Hence, $C \cap C^{\prime}$ is a face of $C$, and similarly, we can see that it is a face of $C^{\prime}$. Finally, suppose that $C, C^{\prime}$ are distinct faces of a single PBW i-linearity domain $P$. Then $C \cap C^{\prime}$ is a face of $C$, and of $C^{\prime}$, since $P$ is a polyhedral cone. We have therefore shown that the set of PBW i-linearity domains, together with all their faces, forms a fan in $\mathbb{R}^{N}$ as required.

\section{5. q-COMMUTING PRODUCTS OF QUANTUM FLAG MINORS}

For any reduced decomposition $\tilde{w}=s_{i_{1}} \ldots s_{i_{k}}$ of an element $w$ in $W$, we define as in [5], see also [2], the quantum flag minors $\Delta_{\tilde{w}}^{*}$. Roughly speaking, $\Delta_{\tilde{w}}^{*}$ is the element of the dual canonical basis corresponding to the extremal vector of weight $w \varpi_{i_{k}}$ in the Weyl module with highest weight $\varpi_{i_{k}}$. By [4, we have:

Proposition 5.1. Fix a reduced decomposition $\mathbf{i}=\left(i_{1}, i_{2}, \ldots, i_{N}\right)$ in $R\left(w_{0}\right)$. Set $\Delta_{k}^{*}=\Delta_{\tilde{w}_{k}}^{*}$, where $\tilde{w}_{k}=s_{i_{1}} \ldots s_{i_{k}}, 1 \leq k \leq N$. Then, the algebra $A_{\mathbf{i}}$ generated by the $\Delta_{k}^{*}$ is a q-polynomial algebra, spanned (as a space) by a part of the dual canonical basis.

Let $d_{\mathbf{i}}$ be the form on $\mathbb{Z}^{N}$ defined by

$$
d_{\mathbf{i}}(\mathbf{m}, \mathbf{n})=\sum_{j<i}\left(\beta_{i}, \beta_{j}\right) m_{i} n_{j}+\sum_{i} m_{i} n_{i}
$$

where the $\beta_{k}$ 's are the roots associated to i. We denote it by $d$ if no confusion occurs. The form $d_{\mathbf{i}}$ encodes the $q$-commutations in the graded algebra $\operatorname{Gr}_{\mathbf{i}}\left(U^{+}\right)$ (see 4]) associated to i. To be more precise, set

$$
\mathbf{n}_{k}=\sum_{t, t \leq k, i_{t}=i_{k}} \mathbf{e}_{t}
$$

Then:

Proposition 5.2. Fix $\mathbf{i}$ in $R\left(w_{0}\right)$. For $k, 1 \leq k \leq N$, we have $\Delta_{k}^{*}=B_{\mathbf{i}}\left(\mathbf{n}_{k}\right)^{*}$. Moreover, for all $\mathbf{m}, \mathbf{n}$ in $\mathbb{Z}_{\geq 0}^{N}$ : 
(i) $q^{d(\mathbf{m}, \mathbf{n})} B(\mathbf{m})^{*} B(\mathbf{n})^{*} \in B(\mathbf{m}+\mathbf{n})^{*}+\sum_{\mathbf{l} \prec \mathbf{m}+\mathbf{n}} \mathbb{Z}\left[q, q^{-1}\right] B(\mathbf{l})^{*}$

(ii) $q^{d\left(\mathbf{n}_{k}, \mathbf{m}\right)} \Delta_{k}^{*} E(\mathbf{m})^{*} \in E\left(\mathbf{n}_{k}+\mathbf{m}\right)^{*}+q \mathcal{L}^{*}$.

Proof: The first assertion is [4, 2.1]. Part (i) is a straightforward consequence of [5. Corollary 2.1 (iii) and Theorem 1.2]. Part (ii) is given by [5, Theorem 3.2].

As in [13, we associate a reduced decomposition $\mathbf{i}(Q)$ (up to commutation) of $w_{0}$ to each quiver $Q$ whose underlying graph is $\mathrm{A}_{n}$. Let $R(Q) \subset R\left(w_{0}\right)$ be the set of all reduced decompositions associated to $Q$.

Lemma 5.3. All quantum flag minors can be realized in the form $B_{\mathbf{i}}\left(\mathbf{n}_{k}\right)$, with $\mathbf{i} \in R(Q)$, for some quiver $Q$ of type $A_{n}$.

Proof: See [5, 4.3].

Remark that if $\mathbf{i}$ is associated to a quiver orientation, then $\prec_{\mathbf{i}}$ is the so-called degeneration ordering, [3], and $d_{\mathbf{i}}$ is Reineke's homological form, see [17. As a consequence, we have:

Proposition 5.4. Let $Q$ be a quiver of type $A_{n}$. Fix a reduced decomposition $\mathbf{i}$ in $R(Q)$. Then, for all $k, 1 \leq k \leq N$,

(i) $d_{\mathbf{i}}\left(\mathbf{n}_{k}\right.$, ?) is increasing for $\prec_{\mathbf{i}}$, i.e. $\mathbf{m} \prec_{\mathbf{i}} \mathbf{n} \rightarrow d_{\mathbf{i}}\left(\mathbf{n}_{k}, \mathbf{m}\right) \leq d_{\mathbf{i}}\left(\mathbf{n}_{k}, \mathbf{n}\right)$,

(ii) $q^{d\left(\mathbf{n}_{k}, \mathbf{m}\right)} B\left(\mathbf{n}_{k}\right)^{*} B(\mathbf{m})^{*} \in B\left(\mathbf{n}_{k}+\mathbf{m}\right)^{*}+q \sum_{\mathbf{l} \prec \mathbf{n}_{k}+\mathbf{m}} \mathbb{Z}[q] B(\mathbf{l})^{*}$.

Proof: (i) is [5, Proposition 4.2]. (ii) follows from (i) together with Proposition 5.2 and Proposition 2.3 (iii).

We can now prove our main theorem.

Theorem 5.5. Let $c$ be a q-commuting product of quantum flag minors and let $b$ be an element of $\mathcal{B}^{*}$ which $q$-commutes with $c$. Then, cb is an element of $\mathcal{B}^{*}$ up to a power of $q$.

Proof: In the sequel, the equalities are given "up to a power of $q$ ". Set $c=$ $b_{m} b_{m-1} \cdots b_{1}$, where the $b_{k}, 1 \leq k \leq m$, are $q$-commuting quantum flag minors. Using an induction on $m$, we know that $c$ is an element of $\mathcal{B}^{*}$ up to a power of $q$.

Now, by Lemma 5.3 for each $k$ we can fix a reduced decomposition $\mathbf{i}_{k}$ in $R(Q)$ (for some quiver $Q$ of type $\mathrm{A}_{n}$ ) associated to the quantum flag minor $b_{k}$. Let $\mathbf{p}_{k}$, resp. $\mathbf{p}$, be such that $b_{k}=B_{\mathbf{i}_{1}}\left(\mathbf{p}_{k}\right)$, resp. $b=B_{\mathbf{i}_{1}}(\mathbf{p})$. We have $c \in q^{\mathbb{Z}} B_{\mathbf{i}_{1}}\left(\sum_{k} \mathbf{p}_{k}\right)$. Hence, by Theorem $3.7 \mathbf{p}$ and $\sum_{k} \mathbf{p}_{k}$ are in a single $\mathbf{i}_{1}$-linearity domain, since $b$ and $c q$-commute. Moreover, this theorem implies also that $\mathbf{p}_{r}, 1 \leq r \leq m$, and $\sum_{k=1}^{s} \mathbf{p}_{k}, 1 \leq s \leq m$ are in a single $\mathbf{i}_{1}$-linearity domain. By Proposition $4.2 \mathbf{p}, \mathbf{p}_{r}$, $\sum_{k=1}^{s} \mathbf{p}_{k}, 1 \leq r, s \leq m$, are in a single $\mathbf{i}_{1}$-linearity domain. Hence :

$$
R_{\mathbf{i}_{1}}^{\mathbf{i}_{s}}\left(\mathbf{p}_{k}+\ldots+\mathbf{p}_{1}+\mathbf{p}\right)=R_{\mathbf{i}_{1}}^{\mathbf{i}_{s}}\left(\mathbf{p}_{k}\right)+\ldots+R_{\mathbf{i}_{1}}^{\mathbf{i}_{s}}\left(\mathbf{p}_{1}\right)+R_{\mathbf{i}_{1}}^{\mathbf{i}_{s}}(\mathbf{p}), \quad 1 \leq k, s \leq m .
$$

By Proposition 5.4 (ii),

$$
B_{\mathbf{i}_{1}}\left(\mathbf{p}_{1}\right)^{*} B_{\mathbf{i}_{1}}(\mathbf{p})^{*} \in B_{\mathbf{i}_{1}}\left(\mathbf{p}_{1}+\mathbf{p}\right)^{*}+q \sum_{\mathbf{l}_{\mathbf{1}} \prec \mathbf{i}_{1} \mathbf{p}_{1}+\mathbf{p}} \mathbb{Z}[q] B_{\mathbf{i}_{1}}\left(\mathbf{l}_{\mathbf{1}}\right)^{*}
$$

Suppose by induction on $k$ that

$$
b_{k} \ldots b_{1} b \in B_{\mathbf{i}_{k}}\left(R_{\mathbf{i}_{1}}^{\mathbf{i}_{k}}\left(\mathbf{p}_{k}+\ldots+\mathbf{p}_{1}+\mathbf{p}\right)\right)^{*}+q \sum_{\substack{\mathbf{l}_{\mathbf{k}} \prec \mathbf{i}_{k} \\ R_{\mathbf{i}_{1}}^{\mathbf{i}_{k}}\left(\mathbf{p}_{k}+\ldots+\mathbf{p}_{1}+\mathbf{p}\right)}} \mathbb{Z}[q] B_{\mathbf{i}_{k}}\left(\mathbf{l}_{\mathbf{k}}\right)^{*} .
$$


Then,

$$
\begin{gathered}
b_{k+1} \ldots b_{1} b \in B_{\mathbf{i}_{k+1}}\left(R_{\mathbf{i}_{1}}^{\mathbf{i}_{k+1}}\left(\mathbf{p}_{k+1}\right)\right)^{*}\left(B_{\mathbf{i}_{k}}\left(R_{\mathbf{i}_{1}}^{\mathbf{i}_{k}}\left(\mathbf{p}_{k}+\ldots \mathbf{p}_{1}+\mathbf{p}\right)\right)^{*}\right. \\
\left.+q \sum_{\mathbf{l}_{\mathbf{k}} \prec \mathbf{i}_{k} R_{\mathbf{i}_{1}} \mathbf{i}_{k}\left(\mathbf{p}_{k}+\ldots+\mathbf{p}_{1}+\mathbf{p}\right)} \mathbb{Z}[q] B_{\mathbf{i}_{k}}\left(\mathbf{l}_{\mathbf{k}}\right)^{*}\right) \\
=B_{\mathbf{i}_{k+1}}\left(R_{\mathbf{i}_{1}}^{\mathbf{i}_{k+1}}\left(\mathbf{p}_{k+1}\right)\right)^{*}\left(B_{\mathbf{i}_{k+1}}\left(R_{\mathbf{i}_{1}}^{\mathbf{i}_{k+1}}\left(\mathbf{p}_{k}+\ldots \mathbf{p}_{1}+\mathbf{p}\right)\right)^{*}\right. \\
+q \sum_{\mathbf{l}_{\mathbf{k}} \prec \mathbf{i}_{k}} \sum_{\mathbf{i}_{1} \mathbf{i}_{k}\left(\mathbf{p}_{k}+\ldots+\mathbf{p}_{1}+\mathbf{p}\right)}^{\left.\mathbb{Z}[q] B_{\mathbf{i}_{k+1}}\left(R_{\mathbf{i}_{k}}^{\mathbf{i}_{k+1}}\left(\mathbf{l}_{\mathbf{k}}\right)\right)^{*}\right) .}
\end{gathered}
$$

In the previous sum, we have, by Proposition 5.2 (i) and $(*)$ :

$$
R_{\mathbf{i}_{k}}^{\mathbf{i}_{k+1}}\left(\mathbf{l}_{\mathbf{k}}\right) \prec_{\mathbf{i}_{k+1}} R_{\mathbf{i}_{1}}^{\mathbf{i}_{k+1}}\left(\mathbf{p}_{k}\right)+\ldots R_{\mathbf{i}_{1}}^{\mathbf{i}_{k+1}}\left(\mathbf{p}_{1}\right)+R_{\mathbf{i}_{1}}^{\mathbf{i}_{k+1}}(\mathbf{p})=R_{\mathbf{i}_{1}}^{\mathbf{i}_{k+1}}\left(\mathbf{p}_{k}+\ldots \mathbf{p}_{1}+\mathbf{p}\right) .
$$

Now, by Proposition 5.4 and the linearity property $(*)$, we obtain the induction.

We can now show that $c b$ satisfies the properties (i) and (ii) of Corollary 2.4 Taking $k=m$, and using Proposition 2.3 and the transitivity of $\prec_{\mathbf{i}_{k}}$, the previous results imply that, up to a power of $q, c b=E_{\mathbf{i}_{m}}\left(R_{\mathbf{i}_{1}}^{\mathbf{i}_{m}}\left(\mathbf{p}_{m}+\cdots+\mathbf{p}_{1}+\mathbf{p}\right)\right)^{*}+q x$, where $x$ is a $\mathbb{Z}[q]$-linear combination of dual PBW-basis elements $E_{\mathbf{i}_{m}}(\mathbf{n})^{*}$ with $\mathbf{n}<R_{\mathbf{i}_{1}}^{\mathbf{i}_{m}}\left(\mathbf{p}_{m}+\cdots+\mathbf{p}_{1}+\mathbf{p}\right)$, and therefore $c b$ satisfies property Corollary [2.4](ii). Now, Part (i) of Corollary 2.4 is clear because $c$ and $b$ are $q$-commuting elements of the dual canonical basis.

This implies that the answer to Question 1.1 is yes when the real element is in the adapted algebra associated to any reduced decomposition.

Corollary 5.6. Let $\mathbf{i}$ in $R\left(w_{0}\right)$ and suppose that b in $\mathcal{B}^{*}$ and $c$ in $\mathcal{B}^{*} \cap A_{\mathbf{i}} q$-commute. Then, $b$ and $c$ are multiplicative.

\section{REFERENCES}

[1] A. Berenstein and A. Zelevinsky. String bases for quantum groups of type $A_{r}$. I. M. Gelfand Seminar, 51-89, Adv. Soviet Math. 16, Part 1, Amer. Math. Soc., Providence, RI, 1993.

[2] A. Berenstein and A. Zelevinsky. Tensor product multiplicities, canonical bases and totally positive varieties. Invent. Math., 143 (2001), 77-128.

[3] K. Bongartz. On degenerations and extensions of finite-dimensional modules. Adv. Math., 121, (1996), no. 2, 245-287.

[4] P. Caldero. Adapted algebras for the Berenstein-Zelevinsky conjecture. To appear in Transformation Groups.

[5] P. Caldero. A multiplicative property of quantum flag minors. To appear in Representation Theory.

[6] R. W. Carter and R. J. Marsh. Regions of linearity, Lusztig cones and canonical basis elements for the quantized enveloping algebra of type $A_{4}$. J. Algebra 234, no.2, 545-603, 2000.

[7] A. Joseph. Quantum groups and their primitive ideals. Springer-Verlag, 29, Ergebnisse der Mathematik und ihrer Grenzgebiete, 1995.

[8] M. Kashiwara. On crystal bases of the $q$-analogue of universal enveloping algebras. Duke Math. J., 63(2), 465-516, 1991.

[9] M. Kashiwara. The crystal base and Littelmann's refined Demazure character formula. Duke Math. J., 71(3):839-858, 1993.

[10] B. Leclerc. Imaginary vectors in the dual canonical basis of $U_{q}(\mathfrak{n})$. Preprint arXiv:math.QA/0202148 2002.

[11] B. Leclerc, M. Nazarov and J.-Y.Thibon. Induced representations of affine Hecke algebras and canonical bases of quantum groups. Preprint arXiv:math.QA/0011074 2000.

[12] S. Z. Levendorskii and Y. S. Soibelman. Some applications of quantum Weyl group. J. Geom. Phys., 7 241-254, 1990. 
[13] G. Lusztig. Canonical bases arising from quantized enveloping algebras. J. Amer. Math. Soc. 3, no. 2, 447-498, 1990.

[14] G. Lusztig. Introduction to quantum groups. Progress in Mathematics, 110, Birkhäuser, 1993.

[15] G. Lusztig. Tight monomials in quantized enveloping algebras. In: Quantum deformations of algebras and their representations, Israel Math. Conf. Proc., Vol. 7, 117-132, 1993.

[16] T. Nakashima and A. Zelevinsky. Polyhedral realizations of crystal bases for quantized KacMoody algebras. Adv. in Math., 131, no. 1, 253-278, 1997.

[17] M. Reineke. Multiplicative properties of dual canonical bases of quantum groups. J. Algebra 211, 134-149, 1999.

[18] M. Rosso. Analogues de la forme de Killing et du théorème de Harish-Chandra pour les groupe quantiques. Ann. Sci. Ed. Norm. Sup., 23, 445-467, 1990.

[19] Y. Saito. PBW basis of quantized enveloping algebras. Publ. Res. Inst. Math. Sci., 30, 209$232,1994$.

[20] T. Tanisaki. Killing forms, Harish-Chandra isomorphisms, and universal $R$-matrices for quantum algebras. J. Mod. Phys. A, Vol. 7, Suppl. 1B, 941-961, 1992.

[21] N. Xi. Canonical basis for type $A_{3}$. Comm. Algebra 27, No. 11, 5703-5710, 1999.

Département de Mathématiques, Université Claude Bernard lyon I, 69622 Villeurbanne Cedex, France

E-mail address: caldero@igd.univ-lyon1.fr

Department of Mathematics and Computer Science, University of Leicester, UniverSITY ROAD, LEICESTER LE1 7RH, ENGLAND

E-mail address: rjm25@mcs.le.ac.uk 